\title{
EDITORIAL
}

\section{Acupuncture: Does it Need a Real Relook?}

\author{
M Ravishankar ${ }^{1}$, S Parthasarathy ${ }^{2}$ \\ Keywords: Acupuncture, Analgesia, Anesthesia, Points. \\ SBV Journal of Basic, Clinical and Applied Health Science (2019): 10.5005/jp-journals-10082-02228
}

Acupuncture is a method of treating certain diseases by inserting solid thin needles into various parts of the body. It is derived from two Latin words: acus meaning needle and puncture meaning prick. Originating from China thousands of years ago, it has rooted itself in many Western and a few Asian countries. There are essentially two different concepts about acupuncture. One is the traditional one which describes an energy called Qi flowing through 20 described meridians. Acupuncture points are described in these meridians and needling helps to cure illnesses. The classical yin-yang theory states that yin and yang are of opposite dynamics but together form a whole existing in balance which constitutes a state of harmony and good health. Yin represents slow, cold, or passive aspects, while yang is about hot, excited, or active aspect of a person. An internal imbalance of yin and yang leads to blocked flow of Qi through meridians and a disease later. Qi can be unblocked by needling points connecting these meridians to restore and maintain health. The acquirement of knowledge about such meridians and points of needle insertions were cumbersome for a simple practitioner. With the expanding knowledge of anatomy and physiology, the modern practice of acupuncture, although superficially resembling the traditional acupuncture practiced in ancient China, is actually quite different. The Chinese when inventing acupuncture did not know that muscles existed. The scientific practice of acupuncture, while retaining the points, varied in the approach in a few ways. The moxibustion is not frequently practiced: the material for needles does not matter: the tonification of points is not acceptable: the mechanism of action has been redefined. ${ }^{1}$ Recently, there are a few possible mechanisms of acupuncture being described: a direct stimulation causing increased blood flow and healing, a possible gate control theory, stimulation of endogenous opioids, downregulation of substance $P$, regional changes in cerebral blood flow, and an anti-inflammatory effect. ${ }^{2}$ Acupuncture anesthesia is still used in China for craniotomy and thyroidectomy, ${ }^{3}$ and it is infrequently employed today for open heart operations as it was in the 1970s. The neurobiological basis has been postulated but not clearly established. Pricking a few points in the body to cause enough sedation for a patient to accept an incision looks strange but the fact remains established. There are innumerable varieties of chronic pain syndromes where acupuncture is very effective. Migraine, myofascial pain syndrome, postherpetic neuralgia, and complex regional pain syndrome are few of the many indications where the use of acupuncture has been proved to be very effective. ${ }^{4,5}$ Even an advanced research in medical science clearly cannot establish the pathophysiology of the above indications. This fact actually turns our eye toward an alternate mode like acupuncture. There are many acute pain conditions like postoperative pain and labor pain where it has been found to be useful. Especially when considering labor pain, it has a role in modulating a dysfunctional labor into a favorable one. ${ }^{6}$ There are a wide variety of other indications like addiction, eye
1,2Department of Anesthesiology, Mahatma Gandhi Medical College and Research Institute, Sri Balaji Vidyapeeth Deemed University, Puducherry, India

Corresponding Author: S Parthasarathy, Department of Anesthesiology, Mahatma Gandhi Medical College and Research Institute, Sri Balaji Vidyapeeth Deemed University, Puducherry, India, Phone: +91 9344304042, e-mail: painfreepartha@gmail.com

How to cite this article: Ravishankar M, Parthasarathy S. Acupuncture: Does it Need a Real Relook? J Basic Clin Appl Health Sci 2019;2(3):87-88.

Source of support: Nil

Conflict of interest: None

disorders, Bell's palsy, ${ }^{4}$ and a few respiratory diseases. The technique of acupuncture that is often practiced involves penetrating the skin with thin, solid, metallic needles that are manipulated by either the hands or by electrical stimulation. The size and the type of the needles used along the depth of insertion depend on the acupuncture style being practiced. As the needles are not hollow, the pain on insertion is less than what is thought of. Acupuncture is not without complications. Even though less common, bleeding, local soreness, fainting, nerve damage, transmission of infections, and damage to internal organs have been reported. As acupuncture practice moves toward scientific medical people from quacks, the anatomical knowledge, the awareness about asepsis, and the availability of disposable needles have made complications negligible. A diagnosis-based approach to points has superseded syndromic pricks. An area of skin infection, seizures, patients on pacemaker, and coagulopathy form relative contraindications to this form of treatment. ${ }^{7}$ Percutaneous electrical nerve stimulation ${ }^{8}$ (PENS) is being recently described for many neuropathic conditions in modern allopathic practice. Is PENS a backdoor entrant to satisfy modern practitioners who do not believe in acupuncture, remains a pertinent question. In this scenario of innumerable pain syndromes and the thread of their pathophysiology still unwound, the truth that acupuncture relieves such pain with reliable efficacy has to be accepted by current medical practitioners. To conclude, the real need of the hour is to remove the mental block about acupuncture from our thoughts to practice the specialty more and more. The inclusion of this specialty as a part of training of medical graduates has to be given a serious thought.

\section{References}

1. Wang SM, Zeev NK, White P. Acupuncture analgesia: I. The scientific basis. Anesth Analg 2008;106(2):602-610. DOI: 10.1213/01. ane.0000277493.42335.7b.

2. Zhang R, Lao L, Ren K, Berman BM. Mechanisms of acupunctureelectroacupuncture on persistent pain. Anesthesiology 2014;120(2):482-503. DOI: 10.1097/ALN.0000000000000101.

(c) The Author(s). 2019 Open Access This article is distributed under the terms of the Creative Commons Attribution 4.0 International License (https://creativecommons. org/licenses/by-nc/4.0/), which permits unrestricted use, distribution, and non-commercial reproduction in any medium, provided you give appropriate credit to the original author(s) and the source, provide a link to the Creative Commons license, and indicate if changes were made. The Creative Commons Public Domain Dedication waiver (http://creativecommons.org/publicdomain/zero/1.0/) applies to the data made available in this article, unless otherwise stated. 
3. Hyodo M, Nakamura N, Yukimati T, Ohki J, Yoshida A, Inamori K, et al. The experience of acupuncture anesthesia. Masui Jap J Anesthesiol 1973;22(3):251-258.

4. Wang $H$, Yang $G$, Wang S, Zheng X, Zhang W, Li Y. The most commonly treated acupuncture indications in the United States: a cross-sectional study. Am J Chin Med 2018(07):1-33. DOI: 10.1142/S0192415X18500738.

5. Thirilogasundary MR, Parthasarathy $S$. Use of electro acupuncture in the treatment of complex regional pain syndrome - a case series study. Eur J Biomed Pharma Sci 2015;2:179-181.
6. Ravishankar M, Parthasarathy S, Saravanan P. Labour analgesia. J Anaesth Clin Pharmacol 1995;15:225-252.

7. Xu S, Wang L, Cooper E, Zhang M, Manheimer E, Berman B, et al. Adverse events of acupuncture: a systematic review of case reports. Evid Based Complement Alternat Med 2013;2013:581203. DOI: 10.1155/2013/581203.

8. Perryman LT. Peripheral nerve stimulation and percutaneous electrical nerve stimulation in pain management: a review and update on current status. Int J Pain Relief 2017;1:36-41. 